MRS Advances (C) 2017 Materials Research Society

DOI: $10.1557 / \mathrm{adv} .2017 .463$

\title{
A Tool to Characterize the Electrical Influence of the Thermal and Mechanical Behaviors of Materials of Optics for CPV applications
}

Arnaud Ritou ${ }^{1}$, Olivier Raccurt ${ }^{1}$, Philippe Voarino ${ }^{1}$, Pierre Besson ${ }^{2}$ and Mathieu Baudrit ${ }^{1}$

${ }^{1}$ Univ. Grenoble Alpes, INES, F-73375 Le Bourget du Lac, France

CEA, LITEN, Departement des Technologies Solaires, 73375 Le Bourget Du Lac, France

${ }^{2}$ Fraunhofer Chile Research, Av. Vicuña Mackenna 4860, Santiago, Chile

\begin{abstract}
Concentrating Photovoltaics (CPV) field aims to integrate expensive high efficiency multi-junction cells into modules with low cost concentrating optics. The choice of the optics depends on different factors: easiness of fabrication and integration process, added costs, optical efficiency and the profile of the spot uniformity reaching the cell. Indeed, previous work has shown a dependence between electrical performance and spectral and spatial uniformities of the light on the cell. To analyze it, a solar CPV test bench is developed at CEA-INES facilities. Lens and cell temperature can be applied separately, in order to evaluate independently different test conditions, while electrical or optical parameters are recorded. The present work shows how temperature and mechanical variations on first stage concentrating optic affects module performances. Several optics and materials are compared, in order to present the tool capabilities.
\end{abstract}

\section{INTRODUCTION}

Commercial multi-junction cells surpass $40 \%$ efficiency under Concentrated Standard Test Conditions (CSTC). Once integrated into a concentrator photovoltaic (CPV) module, the efficiency of the device drops when tested under real conditions. Module efficiency of $39.8 \%$ CSTC has been recently achieved with the advancement of quadruple-junction solar cells $(\approx 46 \%)$ and achromatic lens [1]. However, most commercial modules equipped with triplejunction cells present efficiency values closer to $35 \%$ in 2015. Different sources of losses cause this efficiency drop between cell and module. In particular, real condition differ from standard test conditions with temperature and illumination variation. Besides inherent manufacturing defects, different losses linked to the optics are added such as reflection and non-uniformities [2]. When designing and optimizing a CPV test bench, attention is turned towards concentrating optics and cell electrical performance.

Electrical behavior of triple junction cell is sensitive to spectrum and light distribution. Under non-optimal spectrum, there is a current mismatch between subcells resulting in current generation limitations. Spatial non-uniform distribution will increase the effect of series resistance and spectral non-uniform distribution will cause spatial limitation of current resulting in lateral current flow [3]. These two phenomenon are inherent to refractive optics mainly used for commercial modules, and will depend on :

The optical components : Different design choice, such as concentration ratio, secondary optical element, optics shape, or material, will impact the light distribution on the cell.

Focal distance : The position of the cell in the module is a main optimization parameter Optics temperature : The temperature will cause material refractive index variations and will distort lens shape. Consequently focal distance and spot shape will change, impacting current generation [4], [5]. 
A test method that measures spectrally resolved irradiance distribution for CPV optical systems is presented [6]. In conjunction with electrical I-V curves it is a mean of visualizing and characterizing the effects of chromatic aberration and non-uniform flux profiles under controllable testing conditions putting in relief the role of thermal expansion for different material constituting the optics. The indoor test bench, METHOD (Measurement of Electrical, Thermal and Optical Devices), decouples the temperatures of the primary optical element (POE). In varying the temperature of the POE, the effects on electrical efficiency, focal distance, spectral sensitivity, acceptance angle and multi-junction current matching profiles can be quantified. Moreover, the tool is integrated to a commercial CPV solar simulator which usually provides only I-V chracateristics.

\section{EXPERIMENTAL SETUP}

\section{Technical description of the test bench}

METHOD is integrated into the solar simulator Helios3198 [7] and takes advantage of the collimated flash light delivered by a Xenon Lamp and a parabolic mirror. In reference to Figure 1., the METHOD is composed of two parts. The 'optical measurement position' acquires the image profile of the spot and the 'electrical measurement' contains an xyz plate mounted to a Peltier module that will host the cell to I-V test. Switching between these two positions is possible thanks to a sliding thermal chamber containing the lens. Main parameters of interest can be controlled and studied independently: lens temperature, cell temperature, cell position, or alignment of the single-lens set-up. In reference to Figure 2., the individual components and their main characteristics can be viewed.
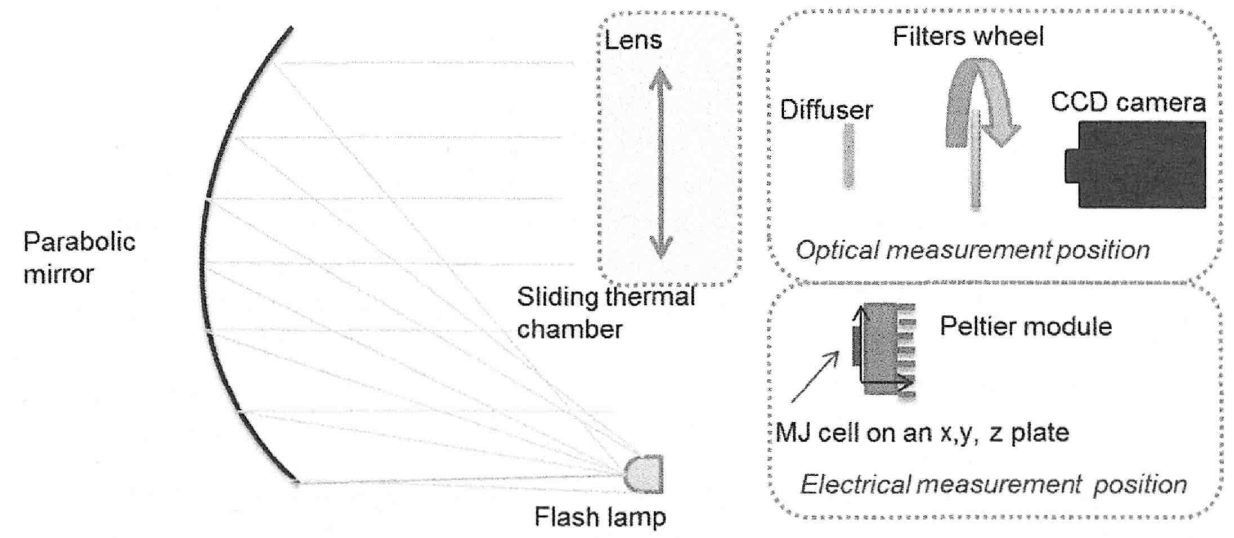

Figure 1. Schematic representation of the METHOD test bench.

The thermal chamber is enclosed by two glass windows and two compact fan heaters placed on each side. Tests have been performed using an infrared camera and thermocouple to verify lens temperature homogeneity. It shows that a good uniformity of lens temperature is only possible if lens is heated at a temperature superior to the temperature expected. Once heaters are turned off, the lens will gradually cool down and temperature uniformity of $2^{\circ} \mathrm{C}$ is ensured. 
During measurements, thermocouples are installed on the inactive part of the optic (glass, side), and allows measuring the temperature as well as verifying the uniformity.

The glass of the thermal chamber modifies incident light on the cell. First, the glass will reflect roughly $4 \%$ of the incident light rays at each optical interface, resulting in a theoretical decrease of $16 \%$ of light intensity compared to a system without glass layers. Then from a spectral point of view, the glass changes the spectrum of the flash. To limit this effect, solar quality glass is used with high transmission more than $90 \%$ in the region $340-1400 \mathrm{~nm}$. For wavelengths inferior to $340 \mathrm{~nm}$, high absorption region of glass impact on the measure is limited as light spectrum and cell spectral response are also weak. A simple manner can be used to address both of these issues: 2 similar glass layers are placed in front of the reference sensors (irradiance sensor and spectral heliometer) of the solar simulator Helios3198. By applying this procedure, single lens module and reference sensors are subject to the same modification of light. Observations of advanced electrical performances showed that glass does not disturb the concentrating optical characterization.

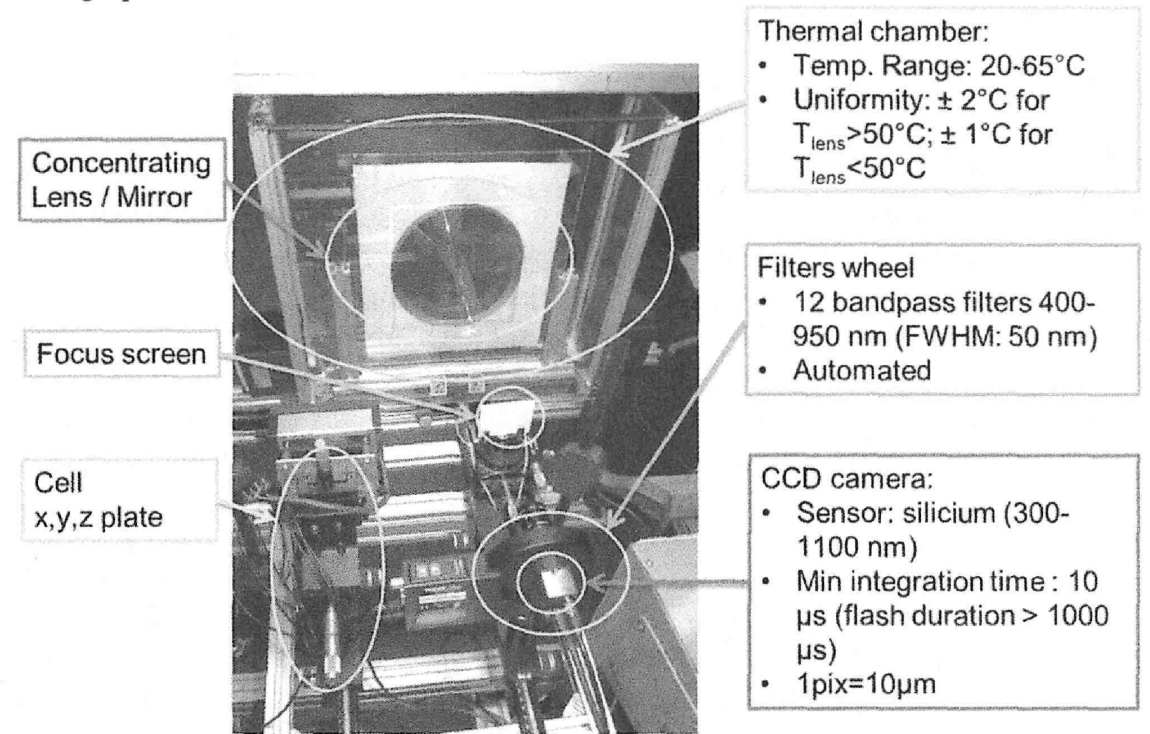

Figure 2. METHOD set-up components and characteristics

The 'optical measurement position' is composed of a bench with micrometric mechanical deck adjustment, an opal diffuser in the focal plane of the optic tested, a filter wheel and a CMOS camera. This setup allows a perfect alignment of the concentrating optic and the optical imaging system (Diffuser + Filter Wheel + Camera). The CMOS silicon camera is coupled to the automated filter wheel composed of 12 filters covering the spectral range from 375 to $975 \mathrm{~nm}$ with a Full Width at Half Maximum (FWHM) of $50 \mathrm{~nm}$ per filter. By imaging the opal diffuser, the camera acquires the light focused by the POE at relatively limited spectral ranges and with the typical outdoor operating temperatures. The CMOS camera's spectral response is comprised between 300 and $1100 \mathrm{~nm}$. This wavelength range corresponds only to the 'Top' and 'Middle' subcells of triple-junction generally used in CPV system tested in our lab. The impact of this issue is limited: indeed, electrically speaking, the photogenerated current of a conventional 
lattice matched triple-junction cell is limited by the 'Top' or 'Middle' subcell. So, it is assumed that leaving 'Bottom' optical information unknown is allowed in CPV module analysis [8].

The 'electrical measurement' composed of 4-wire electrical connections and a Peltier module provides an I-V curve (20 points per curve). The Concentrated Standard Test Condition $(\mathrm{CSTC})$ are given for $1000 \mathrm{~W} / \mathrm{m}^{2}$ irradiance flux and $\mathrm{AM} 1.5 \mathrm{D}$ spectrum at $25^{\circ} \mathrm{C}$. To manage the flash light irradiance, a reference CPV module associated with a spectropyrheliometer (also known as tritube), indicates respectively incident light flux and spectral distribution in the measurement plane area.

\section{RESULTS AND DISCUSSIION}

The METHOD CPV test bench allows us to analyze concentrating optics behavior regarding thermal variation or mechanical displacement. Several studies were done with various optics which are separated here into 2 main categories, refractive and reflective optics. Results of each category is presented in the 2 following paragraphs.

\section{$\underline{\text { Lens analysis }}$}

First, to analyses refractive optics behavior in thermal and mechanical disturb, the optical bench must be perfectly aligned with the source and the displacement axe of focal mechanical decks must be merged with the optical axe. When optical axes are fixed and aligned, irradiance map and I-V curve are recorded for various focal position and temperature.

Here, two different lenses performances are compared against thermal and mechanical parameters. The first lens is a Silicone-on-Glass (SoG) Fresnel lens with a focal length of $285 \mathrm{~mm}$, the second is a PMMA Fresnel lens with $190 \mathrm{~mm}$ focal length. The concentration ratio of each lens is comparable ( $376 \mathrm{X}$ for the SoG one and $404 \mathrm{X}$ for the other). Figure 3. and Figure 4. represent the electrical efficiency recorded on the same $1 \mathrm{~cm}^{2}$ triple-junction cell versus temperature and position on the focal axis.

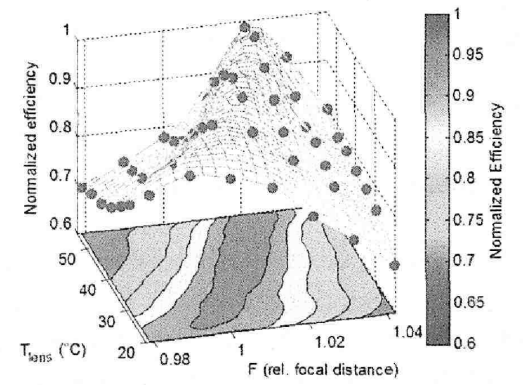

Figure 3. SoG lens electrical efficiency versus temperature and focal distance

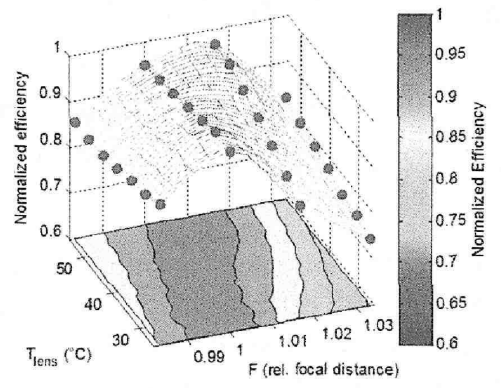

Figure 4. PMMA lens electrical efficiency versus temperature and focal distance

On both figures, the position on optical axis is normalized by the focal length of the lens to compare the same temperature impact on efficiency. On each figure, the maximum efficiency of the cell represent the focal point of the lens and this maximum is taken as the reference. The $\mathrm{Z}$-axis is the Concentrator efficiency divided by the maximum. The focal point is varying along 
the axis linearly with the temperature. Comparing both lenses, we note that PMMA focal point is less affected by the temperature than the SoG one.

\section{Mirror analysis}

For this study, the optical axis is not linear like it is with refractive lenses. To determine position of mirror axis, mirror concentrating behavior is simulated in a ray-tracing software which allows to represent irradiance map seen in different position on the main ray axis. Figure 5. shows the optical axis determined by ray-tracing with the 3 positions of irradiance distribution recording. Figure 6 . is the 3 corresponding irradiance maps recorded.These figures seen on irradiance map are deformed if the measurement axis is not merged to the optical axis. With the optical spot imaging module of METHOD, we adjust the measurement axis angle until irradiance map measured are similar to irradiance map simulated.

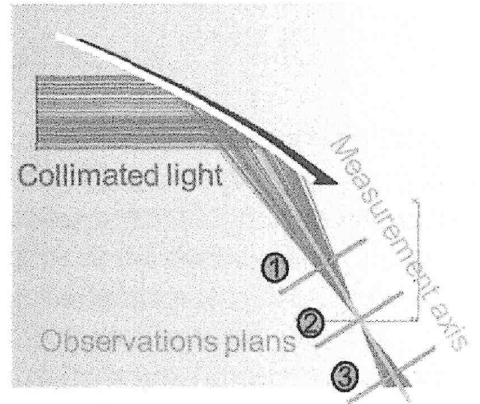

Figure 5. Schematic

configuration of the mirror, the measurement axis and the observation plans with TracePro modelling.

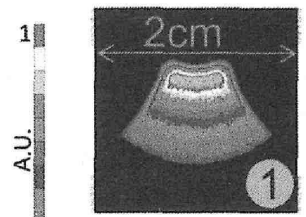

$11.5 \mathrm{~mm}$ before focus point

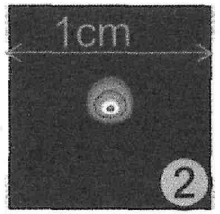

$0 \mathrm{~mm}$ on focus point

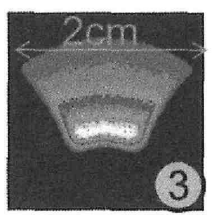

$17.9 \mathrm{~mm}$ after focus point

Figure 6. Spot diagram of the focused light along the measurement axis for three observation plans, situated before, on and after the focus point.

Raw images of spot irradiance map recorded by the CMOS camera are corrected. Opal diffuser, the Filter wheel and the sensor transmission response are taken into account in the measure. The corrected irradiance maps are treated to obtain the spot barycenter also known as centroid. In this case of measurement, 1 pixel represent exactly $10 \times 10 \mu \mathrm{m}^{2}$. The evolution of centroid position versus temperature is given in Figure 7. at various position closed to the focus point. Here, the temperature distorts the mirror. So the focus point is moving in 3D space like it is represented in this graph. On another hand, the light density in the focal plane is quantified and the current density is calculated from the cell's quantum efficiency. Compared to the IV curve computed in the same solar simulator, deeper studies of these non-uniformities and their impact on cell behavior are managed thanks to this tool. 


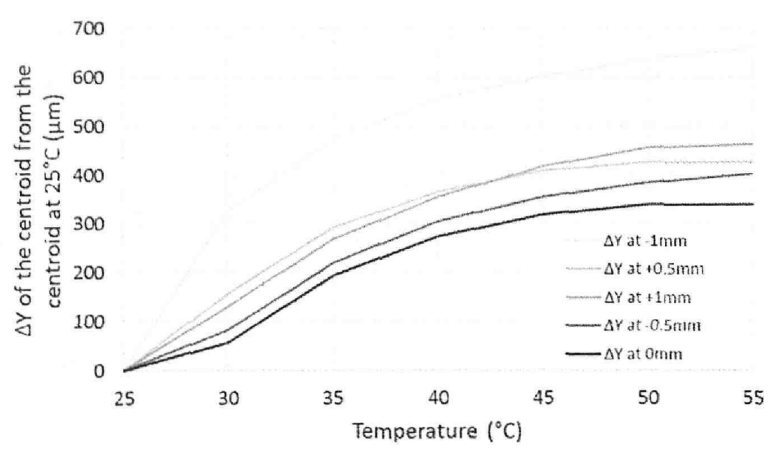

Figure 7. Variation of the $\mathrm{Y}$ coordinates of the centroid versus the temperature for five locations along the measurement axis compared to the centroid's coordinates at $25^{\circ} \mathrm{C}$.

\section{CONCLUSIONS}

CEA has developed a specific bench able to reproduce outdoors real operating conditions of cells and optics in order to measure their performances, electrically and optically. It can be used for quantifying the effect of focal distance and device temperature on the single optical setup performance. Therefore it is a useful tool for an optimization of the module configuration (e.g. cell position) for CSTC and CSOC (optimization in power or in energy). Results presented show how imaging the light irradiance map onto the cell for different wavelength ranges can help understanding electrical behavior of the cell. Effects of non-uniformity (spectral and spatial) on main electrical characteristics Isc fill factor or effective series resistance can be analyzed.

\section{REFERENCES}

1. Current status of CPV technology (2016) Available at: https://www.ise.fraunhofer.de/de/veroeffentlichungen/veroeffentlichungen-pdfdateien/studien- und-konzeptpapiere/current-status-of-concentrator-photovoltaic-cpvtechnology-in-englischer-sprache.pdf. (Accessed: 04 April 2016).

2. R. Herrero, M. Victoria, C. Domínguez, S. Askins, I. Antón, and G. Sala, in AIP Conference Proceedings, vol. 1679, p. 50006 (2015).

3. M. Victoria, R. Herrero, C. Domínguez, I. Antón, S. Askins, and G. Sala, Prog. Photovolt. Res. Appl., vol. 21, no. 3, pp. 308-318,(2013).

4. T. Hornung, M. Steiner, and P. Nitz, Sol. Energy Mater. Sol. Cells, vol. 99, pp. 333-338, (2012).

5. Hornung, T., Bachmaier, A., Nitz, P., \& Gombert, A., AIP Conf. Proc., vol. 1277, no. 1, pp. 85-88, (2010).

6. P. Besson et al., Opt. Express, vol. 24, no. 2, p. A397, (2016).

7. I. Antón, C. Domínguez, M. Victoria, R. Herrero, S. Askins, and G. Sala, Jpn. J. Appl. Phys., vol. 51, no. 10S, p. 10ND12, (2012).

8. P. Besson, PhD of Université de Lyon, (2016). 